\title{
Orientational manoeuvres in the dark: dissociating allocentric and egocentric influences on spatial memory
}

\author{
Neil Burgess ${ }^{\mathrm{a}, \mathrm{b}, *}$, Hugo J. Spiers ${ }^{\mathrm{c}}$, Eleni Paleologou ${ }^{\mathrm{b}}$ \\ anstitute of Cognitive Neuroscience, University College London, Alexandra House, \\ 17 Queen Square, London WIN 3AR, UK \\ ${ }^{b}$ Department of Anatomy and Developmental Biology, University College London, \\ Gower Street, London WC1E 6BT, UK \\ ${ }^{\mathrm{c}} M R C$ Cognition and Brain Sciences Unit, 15 Chaucer Road, Cambridge CB2 2EF, UK
}

Received 18 July 2003; revised 17 November 2003; accepted 19 January 2004

\begin{abstract}
Subjects in a darkroom saw an array of five phosphorescent objects on a circular table and, after a short delay, indicated which object had been moved. During the delay the subject, the table or a phosphorescent landmark external to the array was moved (a rotation about the centre of the table) either alone or together. The subject then had to indicate which one of the five objects had been moved. A fully factorial design was used to detect the use of three types of representations of object location: (i) visual snapshots; (ii) egocentric representations updated by self-motion; and (iii) representations relative to the external cue. Improved performance was seen whenever the test array was oriented consistently with any of these stored representations. The influence of representations (i) and (ii) replicates previous work. The influence of representation (iii) is a novel finding which implies that allocentric representations play a role in spatial memory, even over short distances and times. The effect of the external cue was greater when initially experienced as stable. Females out-performed males except when the array was consistent with self-motion but not visual snapshots. These results enable a simple egocentric model of spatial memory to be extended to address largescale navigation, including the effects of allocentric knowledge, landmark stability and gender. (C) 2004 Elsevier B.V. All rights reserved.
\end{abstract}

Keywords: Spatial updating; Visual snapshot; Viewpoint; Frame of reference; Ideothetic; Landmark

\footnotetext{
* Corresponding author. Address: Institute of Cognitive Neuroscience, University College London, Alexandra House, 17 Queen Square, London W1N 3AR, UK. Tel.: +44-20-7679-1147; fax: +44-20-7679-1145.

E-mail address: n.burgess@ucl.ac.uk (N. Burgess).
}

0022-2860/\$ - see front matter ㄷ 2004 Elsevier B.V. All rights reserved. doi:10.1016/j.cognition.2004.01.001 


\section{Introduction}

How do we remember the locations of objects in the world? Previous experiments using the spatial updating paradigm (Simons \& Wang, 1998; Wang \& Simons, 1999) have illustrated the presence of two types of representation in memory for the locations of objects in an array. One acts like a visual snapshot of the array ${ }^{1}$ that can best be used when the array is in the same configuration relative to the viewer as it was at presentation. The second is an egocentric representation of locations that can be internally updated to accommodate self-motion. Wang and Spelke (2002) have recently argued that these two types of representation, together with a process by which disoriented subjects can re-orient themselves (see Hermer \& Spelke, 1994), are sufficient to provide the cognitive basis for human spatial memory and navigation. This model also appears consistent with evidence for automatic updating of locations to accommodate self-motion (Farrell \& Robertson, 1998; Farrell \& Thomson, 1998; Presson \& Montello, 1994; Rieser, 1989) as well as results indicating a cumulative updating process in tasks where the subject's memory is tested from a different viewpoint than that presented (Diwadkar \& McNamara, 1997; Easton \& Sholl, 1995; Wraga, Creem, \& Proffitt, 2000). It is a simple and clear example of the theoretical position that memory for the locations of objects depends solely on egocentric representations (Roskos-Ewoldsen, McNamara, Shelton, \& Carr, 1998; Scholkopf \& Mallot, 1995; Wang \& Spelke, 2000).

In a classic spatial updating experiment (Wang \& Simons, 1999), following Rieser (1989), subjects view an array of objects on a circular table and, after a brief delay, indicate which one has been moved. Between presentation and test the table or the subject can be moved to provide four conditions: no change, subject rotation, table rotation, subject \& table rotation in the same direction. Although not presented in this way, the experiment can be thought of as a $2 \times 2$ factorial design in terms of the consistency of the test array with visual snapshots or egocentric representations that are internally updatable by self-motion. The test array is consistent with visual snapshot representations in conditions no change and subject\&table because subjects see the same view of it at presentation and test, and consistent with updatable egocentric representations in conditions no change and subject because the array maintains its position relative to an inertial frame of reference. Performance in condition table (inconsistent with both types of representation) can be considered as a baseline above which performance is raised by the effects of consistency with visual snapshot or updatable egocentric representations, assuming that the more appropriate of the two representations will be used where they conflict (as in, e.g. the selection of sensory or motoric representations of path Lambrey, Viaud-Delmon, \& Berthoz, 2002). Improved performance was found when the test array would be consistent with updatable egocentric representations or with visual snapshots, and the effect was greater for the former type of representation. See Fig. 1.

However, there is evidence that spatial behaviour in larger-scale environments can be influenced by external landmarks (Acredolo, Pick, \& Olsen, 1975; Herman \& Siegel, 1978; Jacobs, Thomas, Laurance, \& Nadel, 1998; Mallot \& Gillner, 2000; Presson, 1987;

\footnotetext{
1 This type of representation is also referred to as the 'retinal projection' of the array (Simons \& Wang, 1998; Wang \& Simons, 1999). We prefer 'visual snapshot' because of the lack of visual fixation.
} 

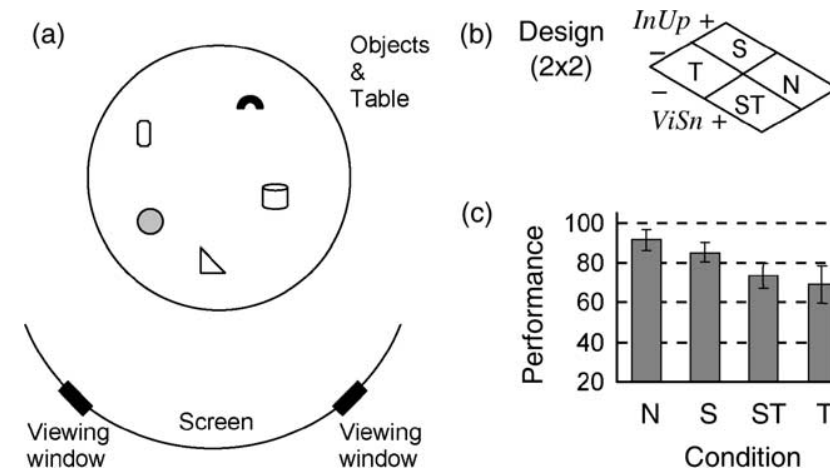

(c)

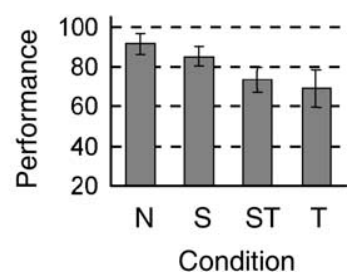

Fig. 1. The spatial updating paradigm of Wang and Simons (1999). (a) Subjects were presented with an array of five objects on a circular table top for $3 \mathrm{~s}$. After a $7 \mathrm{~s}$ delay, during which the table was obscured and one of the objects was moved to a new position, subjects indicated which object had moved. Objects could occupy one of the nine locations in an irregular array. Between presentation and test the table or the subject's viewpoint could be rotated by 47 degrees about the centre of the table, to provide four conditions: no change $(\mathrm{N})$, subject rotation $(\mathrm{S})$, table rotation (T), subject \& table rotation in the same direction (ST). In the $\mathrm{N}$ and $\mathrm{T}$ conditions, subjects moved halfway to the other viewpoint and back again to control for the disruptive effect of moving. Subjects were warned that the table would be rotated in conditions T and ST. (b) $2 \times 2$ factorial design: the test array can be consistent $(+)$ or inconsistent $(-)$ with visual snapshot representations $(\mathrm{ViSn})$ or egocentric representations internally updated by self-motion ( $I n U p)$, see also Fig. 3 top. (c) Performance data from 16 subjects each performing 20 trials of each condition, error bars are $95 \%$ confidence intervals. Consistency with both egocentric representations that are internally updatable by self-motion (InUp) and visual snapshot $(V i S n)$ representations aids performance, with the effect of consistency with updatable egocentric representations being greater than the effect of consistency with visual snapshots. Redrawn from Wang and Simons (1999) experiment 1.

Waller, Loomis, Gollege, \& Beall, 2000; Tlauka \& Wilson, 1994). The association of object locations to external landmarks corresponds to world-centred or 'allocentric' representations rather than egocentric ones. These representations have also been proposed to underlie some of the effects found in memory following shifts of viewpoint (Huttenlocher \& Presson, 1979), and recent experiments have shown increased accuracy when memory is probed from a novel viewpoint aligned with environmental landmarks (McNamara, Rump, \& Werner, 2003) or with the walls of the testing room (Mou \& McNamara, 2002) compared to mis-aligned viewpoints. In addition, effects similar to those attributed to internal updating during a movement of viewpoint can occur in the absence of internal signals relating to actual motion (e.g. vestibular or proprioceptive), as in purely visual virtual reality (Christou \& Bulthoff, 1999) and after imagined movement of viewpoint (Easton \& Sholl, 1995; Wraga et al., 2000).

Are representations of object location relative to external landmarks used in the spatial updating paradigm as well as visual snapshot and updatable egocentric representations? In Wang and Simons' (1999) study the experimental testing room was visible behind the table. Whenever the test array is consistent with updatable egocentric representations (conditions no change and subject) it does not move with respect to the room and so would also be consistent with any representations of object location relative to the external visual cues in the room. Three previous experiments by Simons and Wang (1998) relate to the question of whether environmental cues contribute to their effect of consistency with 


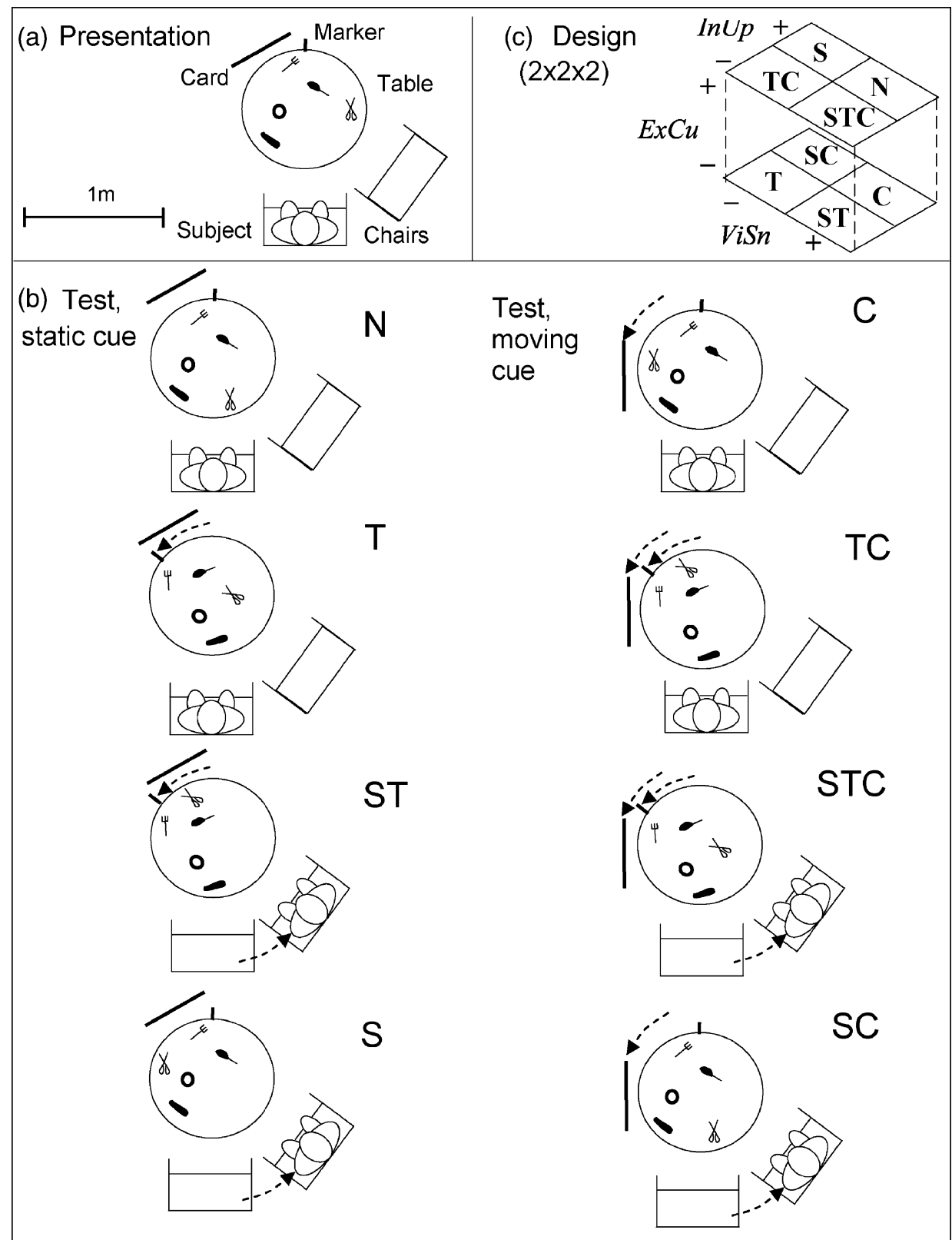

Fig. 2. Experimental apparatus and conditions. (a) Presentation: Plan view of the experimental set up for an example trial in the presentation phase, surrounding black curtains not shown. The objects occupy 5 of the 9 possible locations on the table, the marker is not seen by the subject. (b) Test: Examples of the set up for each of the corresponding conditions following the example presentation trial in (a). The scissors have been moved, shown occupying the four possible remaining locations in the various trials. The letters refer to the experimental conditions, indicating what has been rotated relative to the presentation phase in (a) (see dashed arrow): no change 
updatable egocentric representations. These examined the effect of consistency of the test array with visual snapshot representations in two groups of subjects. One group remained static between presentation and test, and the other moved between two viewpoints. The first experiment, found an effect of visual snapshot consistency in the static group (performance in condition no change exceeded that in condition table) but not in the moving group (no difference between condition subject\&table and condition subject). See Figs. 1 and 3 for the correspondence between conditions and consistency effects. The second used an unlit room and phosphorescent objects to eliminate visual cues within the room, and found the same pattern of results. The third disrupted the internal updating process in the moving viewpoint group by moving them via a rapidly spinning wheeled chair. The performance of this group now showed an effect of visual snapshot consistency (performance in condition subject\&table exceeded that in condition subject). These results demonstrate an effect of consistency with updatable egocentric representations (counteracting the effect of consistency with visual snapshots in the moving group) that remains in the absence of visual cues (the unlit experiment), and that this is due to self-motion signals that are disrupted by spinning. Nonetheless, these experiments do not rule out the possibility that object locations are also represented relative to visual cues in the environment, and that this contributes to the size of the effect of consistency with updatable egocentric representations. Supporting this possibility, performance in condition subject appeared to be worse in the unlit experiment than it is in their experiments in fully visible rooms.

Here we test for the use of a representation of object locations relative to environmental cues external to the array within the spatial updating paradigm. One difficulty is that subjects always have some idea of the space they are in, whether or not it can be seen. Simply turning out the lights or disorienting the subject would not remove this representation, but would leave the experimenter unsure as to its nature or orientation. Accordingly, we attempted to explicitly manipulate representations that depended on a single visual cue external to the object array, so that they would either be consistent with the test array or not. We performed a spatial updating experiment in an unlit room containing a single moveable phosphorescent visual cue external to the array. We used a $2 \times 2 \times 2$ design to compare the three effects of consistency with updatable egocentric representations, visual snapshots or with the external cue. See Methods and Fig. 2. By darkening the room, using earplugs and other measures, we attempted to reduce the availability of uncontrolled cues from the experimental room. However, to the extent that any uncontrolled (e.g. imagined) representations of the room influence performance, we would expect them to improve performance in conditions in which the table is located consistently relative to the room, beyond the performance expected by the use of the three controlled representations.

$(\mathrm{N})$; cue-card $(\mathrm{C})$; table $(\mathrm{T})$; subject $(\mathrm{S})$; subject\&table (ST); table\&cue-card $(\mathrm{TC})$; subject\&cue-card (SC); subject\&table\&cue-card (STC). Note that clockwise rotations of subject, card and table occur as often as the counter-clockwise rotation shown. (c) $2 \times 2 \times 2$ design: + and - signs indicate, for each condition, the consistency of the test array with the three representations: relative to the external cue $(E x C u)$, egocentric representations internally updatable by self-motion ( $\operatorname{InUp}$ ), and visual snapshots ( $\mathrm{ViSn}$ ). 
A final consideration, raised by the use of a moving cue, concerns the question of landmark stability. We are not aware of previous investigations of landmark stability in humans, but it has a noticeable effect in spatial memory tests in rats. Briefly, rats will search for a location defined by visual landmarks (Biegler \& Morris, 1993; Biegler \& Morris, 1996; Fenton, Arolfo, Nerad, \& Bures, 1994; Lenck-Santini, Muller, Save, \& Poucet, 2002; Maurer \& Derivaz, 2000; O’Keefe \& Conway, 1980; Suzuki, Augerinos, \& Black, 1980), but will not do so if the landmark's position has varied from trial-to-trial (Biegler \& Morris, 1993, 1996; Lenck-Santini et al., 2002). We investigated the effect of cue stability by dividing the conditions into two blocks in which the external cue either remains static throughout or in which it moves between presentation and retrieval on every trial. Subjects experiencing the static block first will perceive the external cue as stable compared to those that experience the moving block first. We also used carefully balanced groups of male and female subjects to be sensitive to any effects of gender on the use of the different spatial representations. See Methods and Fig. 2.

\section{Methods}

Sixteen subjects, 8 males and 8 females, participated in this study with age range 20-26 years (mean 22.3, standard deviation 1.7; male: mean 22.9, SD 2.1; female: 21.6, 0.7). Subjects gave their written informed consent, and each received $£ 7.50$ per hour. Their performance IQ was assessed using the short form of Raven's Advanced Progressive Matrices set 1 (mean score 11.25, SD 1.28; male: mean 11.5, SD 1.5; female: 11.1, 1.0). All participants were tested in agreement with the local ethics committee guidelines.

\subsection{Apparatus}

The experiment was conducted in a room $(2.9 \times 3.1 \mathrm{~m})$ with walls covered by black curtains. The room contained a circular table ( $90 \mathrm{~cm}$ in diameter, $45 \mathrm{~cm}$ above the floor), two chairs (seats $47 \mathrm{~cm}$ high), a large piece of card coated with phosphorescent paint (55 cm high, $27 \mathrm{~cm}$ wide, lower edge $46 \mathrm{~cm}$ above the floor), and five common objects (brush, stapler, cup, tape, scissors) coated with phosphorescent paint and lined underneath with towelling. The objects were placed on five of the nine possible positions in an irregular array on the circular table. The distance between any two positions ranged from 22 to $73 \mathrm{~cm}$. The irregularity of the array ensured that no more than two objects were aligned with the observer throughout the experiment. The distance of the chairs to the middle of the table was $120 \mathrm{~cm}$ and the distance between the back of the chairs $100 \mathrm{~cm}$, with subjects sitting with their backs to each chair's back (as they were asked to do) the viewing angle between the chairs was 49 degrees. See Fig. 2. Subjects wore a blindfold on top of sunglasses and a pair of ear plugs. With the blindfold off and the lights off, the subjects were able to see only the five objects and the card (due to the darkness, sunglasses and lack of dark-adaptation caused by leakage of light through the blindfold between trials). The ear plugs and towelling prevented any auditory cues to object location. 


\subsection{Procedure}

On each trial, the lights were turned off, one experimenter instructed the subject to raise the blindfold and view the array of objects. After $3 \mathrm{~s}$ they were instructed to lower the blindfold. A $13 \mathrm{~s}$ delay then occurred during which the lights were turned on, a second experimenter moved one of the objects and the lights were switched off again. This delay is slightly longer than the $7 \mathrm{~s}$ used by Wang and Simons (1999). Subjects were then instructed by the first experimenter to raise the blindfold, to name the object they thought had moved and to replace the blindfold. The lights were then switched on, the second experimenter marked the answer on a response sheet and prepared the table for the next trial. The first experimenter walked around in the space behind and beside the two chairs attempting to randomly vary the positions from which they spoke, so that their voice did not provide a consistent directional cue. The second experimenter remained hidden behind the curtains whenever the subject's blindfold was raised.

The eight conditions varied as to whether or not the subject, table, or card moved between presentation and test. During the delay period the subject was either instructed to move to the other chair or to move half way and back to the original chair. When the table or card was moved, it was rotated about the centre of the table by the same angle as the viewing angle of the two chairs ( 49 degrees). For all trials in all conditions the subjects were warned whether the table and/or the card would move or not. When more than one of the three factors changed position they all moved in the same clockwise or anti-clockwise direction. Thus if the subject and the table moved the view of the objects would be the same at test as it was at study, while if the card and table moved the object locations would have the same position relative to the card.

Each subject experienced 20 trials of each of the 8 conditions for a total of 160 trials. These trials were split into two sessions of 80 trials, which were administered on separate days (mean number of days between sessions $=4.1$ days). Each session was preceded by four training trials, one for each condition. In one session the card stayed still (consisting of the four conditions no change, subject, table, subject\&table), in the other session the card moved (conditions cue-card, subject\&cue-card, table\&cue-card, subject\&table\&cue-card, see Fig. 2). Trials were arranged into blocks of five trials from the same condition, as in Wang and Simons (1999), with these blocks combined in groups of four consisting of one block from each condition in the session, in pseudo random order.

\subsection{Counterbalancing}

The order of the blocks, the possible card positions and session order were counterbalanced across the subjects, and equal numbers of males and females were assigned to each group. At the end of every block of five trials the subject was moved to the start position for the next set of five trials. Half of the subjects (4 males, 4 females) experienced the static card session first, in which the card remained in a position opposite the middle of the pair of chairs. For trials in which the card moved, it occupied one of the two possible places on the far side of the table from the subject. For half of the subjects (4 males, 4 females, 2 each from the static-card first group) the card could be opposite halfway between the chairs or a position rotated 49 degrees clockwise about the centre of 

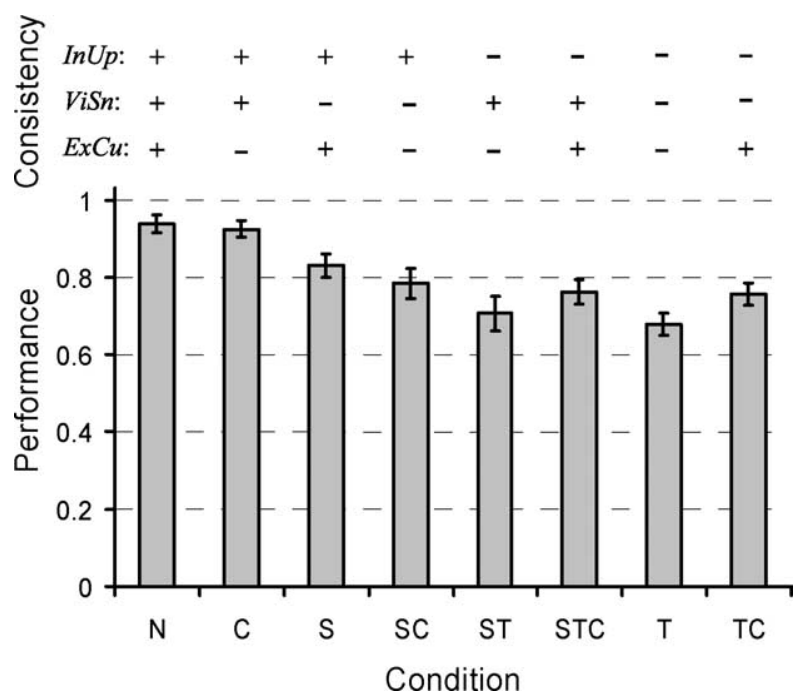

Fig. 3. Performance in the extended spatial updating paradigm. Above: which conditions are consistent with which representations. Below: Columns show the mean performance of the 16 subjects, error bars are the standard error of the mean. Conditions are labelled according to what moves between presentation and test: no change $(\mathrm{N})$; cue-card $(\mathrm{C})$; table $(\mathrm{T})$; subject $(\mathrm{S})$; subject\&cue-card $(\mathrm{SC})$, etc. See Fig. 2 for a diagram explaining the conditions.

the table. For the other half of the subjects, the card could either be in the middle or rotated 49 degrees counter-clockwise. See Fig. 2.

\subsection{Analysis}

Three within-subjects factors were examined in a repeated measures $2 \times 2 \times 2$ ANOVA: consistency of the test array with visual snapshots; consistency with egocentric representations that are internally updatable by self-motion; and consistency with the external cue, see Methods and Figs. 2 and 3. Gender and session order were included as between-subject factors. An $\arcsin$ transform $(\arcsin (\sqrt{ } x))$ was applied to the proportion correct data as several subjects showed levels of performance at or near 1.0 in some conditions (e.g. Winer, Brown, \& Michels, 1991). Significant effects were explored with two-tailed paired sample $t$-tests.

\section{Results}

Significant within-subjects main effects of consistency with all three representations were found. The effect of consistency with visual snapshots was the most significant $(F(1,15)=55.9, \quad P<0.001)$, followed by consistency with internally updatable egocentric representations $(F(1,15)=37.0, P<0.001)$, followed by consistency with the external cue $(F(1,15)=7.4, P<0.02)$. There was a significant interaction between the effects of consistency with visual snapshots and internally updatable egocentric 
representations $(F(1,15)=17.8, P<0.001)$, reflecting the high performance in conditions ( $\mathrm{N}$ and $\mathrm{C}$ ) where both representations were consistent with the test array. There was also a significant interaction between session order and the effects of consistency with the external cue and with updatable egocentric representations $(F=5.1$, $P<0.05)$, and an interaction between gender and visual snapshot consistency $(F=5.1$, $P<0.05)$. There were no other significant interactions or effects of gender and session order.

Performance on the conditions in which the cue was static (no-change, table, subject\&table, subject) closely replicate the pattern of results reported by Wang and Simons (1999), compare Figs. 1c and 3. Performance was better when the table and the subject remained still (condition no change) than when the table moved (condition table; $t(15)=10.9, P<0.001$ ), or the subject moved (condition subject; $t(15)=5.2$, $P<0.001)$. Performance was better when the subject moved alone (condition subject) than when the table moved alone (condition table; $t(15)=4.9, P<0.001$ ) or when the subject and table moved together so that subjects received the same view after moving (condition subject\&table, $t(15)=2.58, P<0.05$ ). When the subject and table moved together the subjects' performance was not significantly better than when the table rotated alone $(t(15)=1.12, P=0.28)$.

The effect of the external cue is shown in better performance whenever the cue and array were consistent compared to inconsistent, i.e. performance in no change $>$ cue-card, subject $>$ subject\&cue-card, table\&cue-card $>$ table, subject\&table\&cuecard $>$ subject\&table, see Figs. $2 \mathrm{c}$ and 3. Of these individual comparisons, only the difference between both table and card moving together (condition table\&cue-card) and the table moving alone (condition table; $t(15)=2.95, P=0.01$ ) achieved statistical significance. This contrast (table \&cue-card - table) is the one corresponding to the single effect of consistency with the external cue in the absence of the other two effects. The size of this single effect on mean performance is quite large at $7.8 \%$, compared to $10.6 \%$ for the single effect of updatable egocentric information (subject\&cue-card - table) and $2.8 \%$ for the single effect of visual snapshot information (subject\&table - table).

\subsection{Interactions with session order and gender}

Since there was a significant interaction between cue consistency, internal updating and session order (i.e. whether the card was experienced as static in the first or second session), we investigated performance as a function of the session in which the subject performed a given condition (Fig. 4). There is a learning effect for most conditions, such that performance is better amongst subjects performing it in their second session. In contrast to this pattern, conditions cue-card and subject\&cue-card show the reverse, i.e. better performance in subjects performing these conditions first. Subjects experiencing the cue card as static in their first session (comprising conditions no change, subject, subject\&table, table) will be more likely to encode object locations relative to the cue in the subsequent moving-cue session (comprising conditions cue-card, subject\&cuecard, subject\&table\&cue-card, table\&cue-card). These subjects would then perform worse in conditions cue-card and subject\&cue-card, as these are the two moving-cue conditions for which locations relative to the cue card are incorrect (the card having moved 


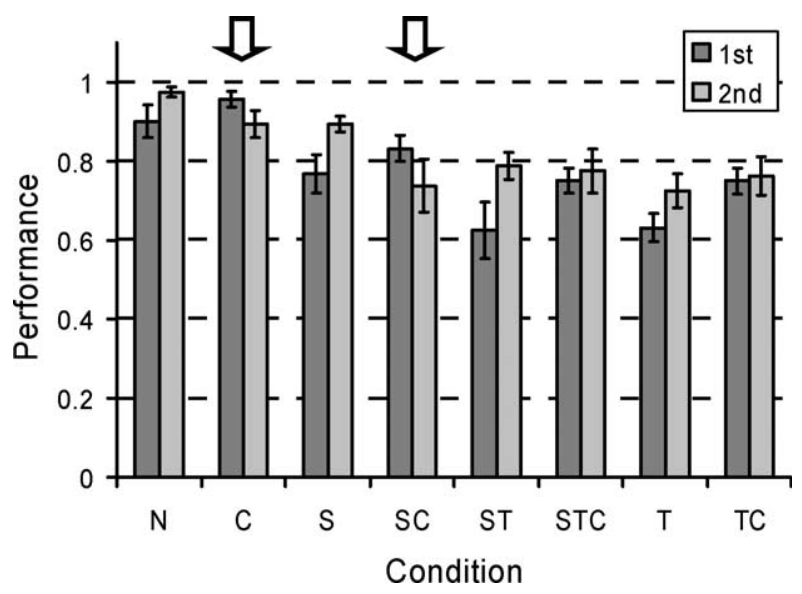

Fig. 4. Performance as a function of session order. Improved performance is seen in the second session compared to the first for all conditions except $\mathrm{C}$ and SC. Columns show the mean performance from the eight subjects experiencing a given condition first (1st) or second (2nd). Note the learning effect in all conditions except for $\mathrm{C}$ and SC (shown by arrows). Error bars are the standard error of the mean. Conditions are labelled according to what moves between presentation and test: no change $(\mathrm{N})$; cue-card $(\mathrm{C})$; table $(\mathrm{T})$; subject $(\mathrm{S})$; subject\&cue-card (SC), etc., see Fig. 2.

relative to the table). We note that a contribution to this effect from a difference between the cue-card-static-first and cue-card-static-second groups cannot be ruled out. In addition, the pattern of performance in other conditions also contributes to the significance of the interaction term found in the ANOVA.

The interaction between gender and visual consistency is largely produced by an overall advantage for females over males (see e.g. the baseline condition table) that is reduced or reversed in the two conditions consistent with updated egocentric representations but inconsistent with visual snapshots (conditions subject and subject\&(ue-card), see Fig. 5.

\section{Discussion}

We showed a significant positive effect of consistency of the orientation of the array of objects with visual snapshots, representations related to external cues and egocentric representations that are internally updatable by self-motion. The effects of consistency with updatable egocentric and visual snapshot representations closely replicate the finding of Wang and Simons (1999). When subjects move around an array (condition subject) their performance is better than when the array rotates with them (condition subject\&table) and is also better than when the array rotates alone (condition table), cf. Figs. 1c and 3. In addition, we found a positive effect of the test array's consistency with an external visual cue. The single effect of consistency with the external cue raises performance when the table and cue rotate together (condition table \& cue-card) above that when the table rotates alone (condition table). The effect of consistency with the external 


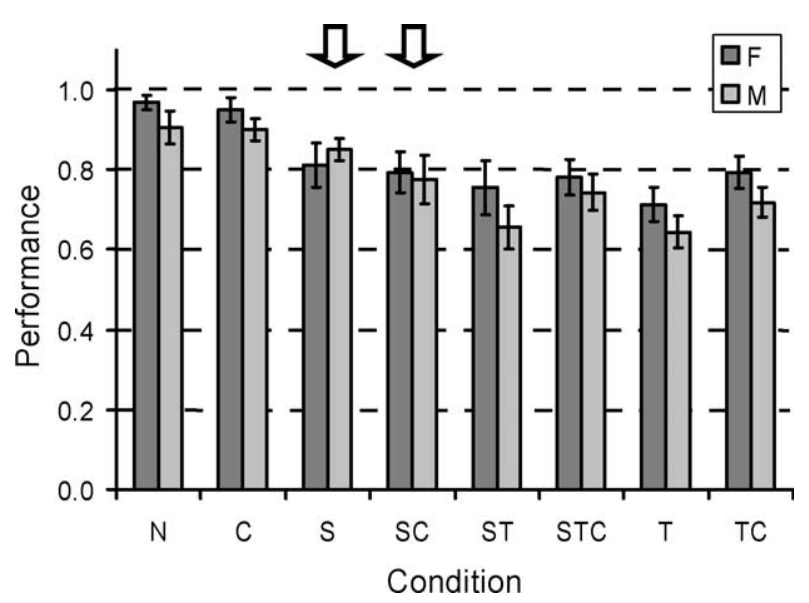

Fig. 5. The effect of gender on performance. In our group of subjects, the females $(\mathrm{F})$ tended to perform better than the males (M) (see e.g. the baseline condition T). This difference tended to be greater in conditions relying on visual snapshot representations (e.g. condition ST), and reduced in those consistent with representations internally updated by the subject's motion (conditions S and SC, shown by arrows). Columns show the mean performance of the eight females (F) and eight males (M). Error bars are the standard error of the mean. Conditions are labelled according to what moves between presentation and test: no change (N); cue-card (C); table (T); subject (S); subject\&cue-card (SC), etc., see Fig. 2.

cue can also be seen in combination with other effects, e.g. when the subject, table, and cue all rotate together (condition subject\&table\&cue-card) the combined effects of consistency with visual snapshots and with the external cue improves performance significantly relative to that when the table rotates alone (condition table; $t(15)=2.58$, $P<0.05$ ), which is not true of the effect of visual snapshot consistency alone (condition subject\&table $; t(15)=1.12, P=0.28)$.

Our results imply that object locations are represented relative to visual landmarks external to the array, even within the short distances and delays present in the spatial updating paradigm. They also imply that part (but certainly not all) of the effect attributed to consistency with updatable egocentric representations in Wang and Simons' (1999) experiment may be due to consistency with the visual landmarks available in the testing room (see also Mou, McNamara, Valiquette, \& Rump, 2004). This is reflected in the reduced significance of the effect of consistency with updatable egocentric representations relative to the effect of consistency with visual snapshots in our experiment compared to that of Wang and Simons (1999), despite our close replication of performance in the conditions present in both experiments. Conversely, our finding of significant effects of consistency with visual snapshots and internally updatable egocentric representations, even when the additional effect of an external cue has been explicitly accounted for, further confirms the presence of these types of representation. Thus Mou et al.'s (2004) opinion that models relying on egocentric representations of familiar views (e.g. Roskos-Ewoldsen et al., 1998; Scholkopf \& Mallot, 1995; Wang \& Spelke, 2000) are no longer tenable given recent results on memory from novel viewpoints does not mean that these representations do not exist (e.g. the 'visual snapshots' in our experiment). 
Simply, there are also other representations, such as those relating objects to environmental cues, which can direct behaviour in appropriate circumstances.

These findings also have a wider significance. The presumed sufficiency of updatable egocentric and visual snapshot representations in the spatial updating paradigm has been used to argue against the presence of environment-centred representations in human memory for locations over the longer distances and times involved in human navigation (Wang \& Spelke, 2002). We therefore suggest that Wang and Spelke's elegantly simple egocentric model requires an additional representation of location relative to environmental landmarks to accommodate our findings within the spatial updating paradigm (see also Mou et al., 2004). This elaboration of their model will also remove an obstacle to its application to behaviour over the longer times and distances involved in real-world navigation. Here the path from the target location (cf. the viewpoint at encoding) to the start location (cf. the viewpoint at retrieval) can extend over miles and days-ruling out accurate movement-related updating, and the target location may be far out of sight of the start location-ruling out the use of visual snapshots. Although the egocentric model allows the use of environmental features to reorient the subject, this process cannot also provide the direction to the target location unless the target location is itself directly represented relative to the environmental features-i.e. unless the external cue-related representation of target locations is added as we are suggesting. Thus, while the presence of visual snapshots and internally updatable egocentric representations is not in doubt, the identification of an additional representation of location relative to the environment is important for understanding the cognitive basis of human navigation.

\subsection{Uncontrolled environmental representations}

The effects of consistency with updatable egocentric, visual snapshot and external cue-related representations are necessary to explain the pattern of performance we observed. But are they sufficient? The super-additive effects of consistency with updatable egocentric representations and consistency with visual snapshots, reflected in the significant interaction between them in the ANOVA, imply that they might not be. The performance advantages for conditions consistent with both representations (i.e. conditions no change and cue-card) relative to the 'baseline' condition table (26 and $25 \%$, respectively) are greater than expected by additively combining the single performance differences due to each effect alone $(10.6 \%$ for updatable egocentric representations, $2.8 \%$ for visual snapshots, $7.8 \%$ for external cue-related). It is possible that these two conditions, in which the positions of both table and subject remains constant within the external room, benefit from additional uncontrolled environmental representations (e.g. an imagined configuration of room walls and door), despite our use of darkness and a controlled environmental cue. A similar pattern can be seen in Simons and Wang's (1998) unlit experiment: the performance advantage for condition no change relative to table exceeds that predicted by adding the effects of consistency with updatable egocentric representations (performance difference subject - table) and consistency with visual snapshots (performance difference subject\&table-table). More generally, whenever the test array is consistent with updatable egocentric representations it will also be consistent with any uncontrolled environmental representations that can 
accommodate self-motion. Thus estimated contributions of egocentric internally updated representations may necessarily include a contribution from uncontrolled environmental representations, even in unlit spatial updating paradigms. ${ }^{2}$

\subsection{Performance of the baseline task and the role of awareness}

Subjects performed well above chance in the 'baseline' task (condition table) in which the updatable egocentric, visual snapshot and external cue-related representations are all inconsistent with the test array. So far we have focused on the modulation of performance caused by consistency with these representations. How did subjects think they performed the basic task? After they had completed both sessions, subjects were asked what method, if any, they had used to solve the task. The most common response related to remembering the shape formed by the object locations (three subjects), or by the locations of various subcategories of object (four subjects). One subject reported remembering objects that formed a triangle and several mentioned remembering any aligned objects. These strategies are consistent with the idea that subjects use frames of reference intrinsic to the array of objects in these types of memory task (Mou \& McNamara, 2002). Beyond this, three subjects reported using verbal codes to remember three of the more central objects, and two subjects reported using a combined verbal and overall shape strategy. It is interesting to note the robustness of the modulatory effects of the consistency manipulations despite the variety of explicit strategies reported.

In addition to being asked how they performed the task overall, subjects were asked if they had used the external cue to facilitate performance. Most subjects said they did not pay any attention to the cue at any point. Only one subject (who did the cue static session first) claimed to have made use of the cue during the majority of the experiment. The significant effect of cue consistency, and the absence of subjects reporting using anything like visual snapshots or updating by self-motion, indicates that subjects are unaware of the processes underlying the modulatory effects of consistency on performance.

\subsection{How does an external cue aid performance?}

We have assumed that the positive effect on performance of consistency with the external cue derives from a representation in which object locations are defined relative to it. It may be that individual object-locations are represented relative to the external cue, as in locations defined relative to a cue card in rat experiments (Lenck-Santini et al., 2002). Alternatively, the orientation of the entire array might be represented relative to the external cue. We cannot distinguish between these alternatives, e.g. we cannot tell whether, in conditions showing an effect of the cue, the size and direction of the effect varies between objects in the array, as only one location is tested. Either explanation would be consistent with the advantage found in recognising scenes from viewpoints aligned with external landmarks (McNamara et al., 2003; Mou \& McNamara, 2002).

\footnotetext{
${ }^{2}$ The alternative is that the three systems mediating the use of snapshot, updatable egocentric and external cue representations are somehow organised such that information in the first two systems, but not the third, combine super-additively when available.
} 
Are there alternative explanations in terms of the other processes already proposed to be at work in the spatial updating paradigm? One alternative is that when the cue rotates with the table, it gives the subject additional information about the size of the rotation. However, Wang and Simons (1999, experiment 2) found no effect on subjects' performance when subjects were allowed to rotate the table themselves compared to when the experimenter rotated it for them. Thus additional knowledge of the table's rotation alone would not be expected to improve performance. The small negative effects of moving the external cue relative to the table (conditions subject\&cue-card versus subject and cue-card versus no change) also go against this interpretation since there is no rotation of the table in these conditions.

A second alternative interpretation is that the external cue is included in the visual snapshot representation and so enhances the visual snapshot effect. However, this is not borne out by the improved performance in condition table\&cue-card relative to table (since both conditions are inconsistent with visual snapshot representations), see Fig. 3 .

A final alternative is that the visual cue enhances the effect of consistency with the updatable egocentric representation, by aiding the updating process. However, this would not predict the positive effect of rotating the cue with the table versus rotating the table alone (condition table \&cue-card versus table), since the test display is inconsistent with any egocentric representations in both conditions. A related interpretation might concern the process of reorientation of updatable egocentric representations relative to the environment, even though this process is thought to apply after disorientation of the subject (Hermer \& Spelke, 1994; Hermer-Vazquez, Spelke, \& Katsnelson, 1999; Wang \& Spelke, 2002). However, for this to produce an effect of consistency with the external cue, the updatable egocentric representation would have to reliably follow the movement of the cue rather than the movement of the subject, even when the subject's viewpoint does not change (as in condition table\&cue-card versus table). Such a representation could not be described as either 'egocentric' or 'internally updated', i.e. this interpretation is equivalent to the proposed representation of location relative to the external cue.

Thus, within the paradigm of explaining performance in terms of consistency with a given type of representation, we must conclude that a representation relative to the external cue is required in addition to egocentric visual representations and egocentric representations updated by self-motion. Alternative ways of analysing and thus interpreting the data are possible, but will generally be less clean, involving separate explanations of performance in the different conditions and representations in which egocentric and allocentric information are mixed.

\subsection{The effect of the external cue's stability}

Subjects performed the various conditions in two sessions such that the external visual cue was stationary relative to the room throughout one session, and moved on every trial (either alone, or with the table or subject or both) in the other session. Although there was no significant main effect of session order, there was an interaction with the main effects of consistency with the external cue and consistency with updatable egocentric representations. In the clearest example of this interaction, a learning effect (i.e. improved performance in the second session compared to the first) was present in all conditions 
except for conditions cue-card and subject\&cue-card (see Fig. 4). In these two conditions the external cue moves relative to the room and to the object array, and performance is better in the first session. One possible interpretation of this is that the effect of the moving cue is stronger (i.e. harder to ignore) in subjects who had previously experienced the cue as static. That is, the effect of a visual landmark may depend on being initially experienced as stable relative to updatable egocentric representations and/or uncontrolled representations of the testing room (the cue is equally unstable across both sessions relative to visual snapshot representations). While we are not aware of experiments on landmark stability in humans, our results are consistent with previous experiments on rats in which the influence of landmarks on searching behaviour depends on their perceived stability (Biegler \& Morris, 1993, 1996; Lenck-Santini et al., 2002).

This finding may relate to experiments in which visual cues are ignored. Since the cue's stability relative to the subject's updatable egocentric representations may affect its influence on behaviour, the influence of a static visual cue might be reduced if the subject's internal sense of direction varies from trial-to-trial. This would explain the finding that when rats are systematically disoriented by spinning before each trial, their search is constrained only by the geometry of the environment, ignoring the visual cues within it (Cheng, 1986) unlike non-disoriented rats (Biegler \& Morris, 1993, 1996; Fenton et al., 1994; Lenck-Santini et al., 2002; Maurer \& Derivaz, 2000; O’Keefe \& Conway, 1980; Suzuki et al., 1980). It might also explain similar findings in the searching patterns of young children (Hermer \& Spelke, 1994) and of adults performing verbal shadowing (Hermer-Vazquez et al., 1999), but cannot explain why non-shadowing adults do use visual landmarks despite having been disoriented.

\subsection{The effect of gender}

The interaction between gender and the effect of visual snapshot consistency, and subsequent inspection of Fig. 5, shows an advantage for females except for the conditions in which the array is inconsistent with visual snapshots but consistent with internally updated egocentric representations (conditions subject and subject\&cue-card). Although care is required in generalising from the very small sample in this study (even though well-matched in age and IQ), the findings are consistent with gender differences found in larger studies. In contrast to mental rotation and spatial navigation which typically reveal an advantage for males over females (Astur, Ortiz, \& Sutherland, 1998; Dabbs, Chang, Strong, \& Milun, 1998; Moffatt, Hampson, \& Hatzipantelis, 1998), females outperform males when asked to identify which object of a set of objects has been moved (McBurney, Gaulin, Devineni, \& Adams, 1997; Silverman \& Eals, 1992)-the basic paradigm used here. The exceptions to this female advantage in conditions subject and subject\&cue-card perhaps indicates an advantage for men in using internal updating that is masked by the advantage for women when visual snapshots can be used. Thus it may be that the noted female preference for recognising landmarks and male preference for using distances, directions and environmental geometry to navigate (Choi \& Silverman, 1997; Galea \& Kimura, 1992; Miller \& Santoni, 1982; Sandstrom, Kaufman, \& Huettel, 1998) relates to lower level differences in the ability to use visual snapshot and updatable egocentric representations. 


\section{Conclusion}

We have demonstrated the importance of visual landmarks in memory for object locations within the spatial updating paradigm. Subjects were significantly better at remembering the array of object locations when it was oriented consistently with respect to a single external visual cue. Thus representations of locations relative to external landmarks play a role in spatial memory even over short distances and times, in addition to the well established contributions of visual snapshot matching and egocentric representations that are internally updated by the subject's self-motion (see also Mou et al., 2004). We could not tell whether it was the individual object-locations or the orientation of the entire array that was related to the external cue. The effectiveness of the external cue depended to some extent on whether subjects initially experienced it to be stable from trial to trial, consistent with experiments in rats. Females performed better than males except for conditions depending on internally updated representations rather than visual snapshots representations. This finding accords with related studies of gender differences in spatial memory, and may relate to the greater dependence on recognising landmarks during navigation in females than in males. Overall, our results indicate a reinterpretation of the spatial updating paradigm, and have wider implications in terms of the cognitive processes available to support human spatial memory and navigation over long distances and times.

\section{Acknowledgements}

We thank Tom Hartley and John O'Keefe for many useful discussions, Frances Wang for communicating details of her previous experiment, and Marko Nardini, Colin Lever, Jon Driver, Iris Trinkler and three anonymous referees for commenting on this manuscript. This work was supported by the Medical Research Council, UK, and by a Wolfson Foundation undergraduate prize to EP.

\section{References}

Acredolo, L. P., Pick, H. L., \& Olsen, M. G. (1975). Environmental differentiation and familiarity as determinants of children's memory for spatial location. Developmental Psychology, 11, 495-501.

Astur, R. S., Ortiz, M. L., \& Sutherland, R. J. (1998). A characterization of performance by men and women in a virtual Morris water task: A large and reliable sex difference. Behavioral Brain Research, 93, 185-190.

Biegler, R., \& Morris, R. G. (1993). Landmark stability is a prerequisite for spatial but not discrimination learning. Nature, 361, 631-633.

Biegler, R., \& Morris, R. G. (1996). Landmark stability: Further studies pointing to a role in spatial learning. Quarterly Journal of Experimental Psychology. B, Comparative and Physiological Psychology, 49, 307-345.

Cheng, K. (1986). A purely geometric module in the rat's spatial representation. Cognition, 23, 149-178.

Choi, J., \& Silverman, I. (1997). Sex dimorphism in spatial behaviors: Application to route learning. Evolution and Cognition, 2, 165-171.

Christou, C. G., \& Bulthoff, H. H (1999). The perception of spatial layout in a virtual world (Rep. No. 75). Tubingen, Germany: Max Planck Institute for Biological Cybernetics. 
Dabbs, J. M., Chang, E. L., Strong, R. A., \& Milun, R. (1998). Spatial ability, navigation strategy, and geographic knowledge among men and women. Evolution and Human Behavior, 19, 89-98.

Diwadkar, V. A., \& McNamara, T. P. (1997). Viewpoint dependence in scene recognition. Psychological Science, 8, 302-307.

Easton, R. D., \& Sholl, M. J. (1995). Object-array structure, frames of reference, and retrieval of spatial knowledge. Journal of Experimental Psychology. Learning, Memory, and Cognition, 21, 483-500.

Farrell, M. J., \& Robertson, I. H. (1998). Mental rotation and the automatic updating of body-centered spatial relationships. Journal of Experimental Psychology. Learning, Memory, and Cognition, 24, 227-233.

Farrell, M. J., \& Thomson, J. A. (1998). Automatic spatial updating during locomotion without vision. Quarterly Journal of Experimental Psychology Section A-Human Experimental Psychology, 51, 637-654.

Fenton, A. A., Arolfo, M. P., Nerad, L., \& Bures, J. (1994). Place navigation in the Morris water maze under minimum and redundant extra-maze cue conditions. Behavioral and Neural Biology, 62, 178-189.

Galea, L. A. M., \& Kimura, D. (1992). Sex differences in route-learning. Personality and Individual Differences, $14,53-65$.

Herman, J. F., \& Siegel, A. W. (1978). The development of cognitive mapping of the large-scale environment. Journal of Experimental Child Psychology, 26, 389-406.

Hermer, L., \& Spelke, E. S. (1994). A geometric process for spatial reorientation in young children. Nature, 370, $57-59$.

Hermer-Vazquez, L., Spelke, E. S., \& Katsnelson, A. S. (1999). Sources of flexibility in human cognition: Dualtask studies of space and language. Cognitive Psychology, 39, 3-36.

Huttenlocher, J., \& Presson, C. C. (1979). The coding and transformation of spatial information. Cognitive Psychology, 11, 375-394.

Jacobs, W. J., Thomas, K. G. F., Laurance, H. E., \& Nadel, L. (1998). Place learning in virtual space: Topographical relations as one dimension of stimulus control. Learning and Motivation, 29, 288-308.

Lambrey, S., Viaud-Delmon, I., \& Berthoz, A. (2002). Influence of a sensorimotor conflict on the memorization of a path traveled in virtual reality. Brain Research. Cognitive Brain Research, 14, 177-186.

Lenck-Santini, P. P., Muller, R. U., Save, E., \& Poucet, B. (2002). Relationships between place cell firing fields and navigational decisions by rats. Journal of Neuroscience, 22, 9035-9047.

Mallot, H. A., \& Gillner, S. (2000). Route navigating without place recognition: What is recognised in recognition-triggered responses? Perception, 29, 43-55.

Maurer, R., \& Derivaz, V. (2000). Rats in a transparent morris water maze use elemental and configural geometry of landmarks as well as distance to the pool wall. Spatial Cognition and Computation, 2, 135-156.

McBurney, D. H., Gaulin, S. J. C., Devineni, T., \& Adams, C. (1997). Superior spatial memory of women: Stronger evidence for the gathering hypothesis. Evolution and Human Behavior, 18, 165-174.

McNamara, T. P., Rump, B., \& Werner, S. (2003). Egocentric and geocentric frames of reference in memory of large-scale space. Psychonomic Bulletin and Review, 10(589), 595.

Miller, L. K., \& Santoni, V. (1982). Sex differences in spatial abilities: Strategic and experimental correlates. Acta Psychology, 53, 1106-1110.

Moffatt, S. D., Hampson, E., \& Hatzipantelis, M. (1998). Navigation in a "virtual" maze: Sex differences and correlation with psychometric measures of spatial ability in humans. Evolution and Human Behavior, 19, $73-87$.

Mou, W., \& McNamara, T. P. (2002). Intrinsic frames of reference in spatial memory. Journal of Experimental Psychology. Learning, Memory, and Cognition, 28, 162-170.

Mou, W., McNamara, T. P., Valiquette, C. M., \& Rump, B. (2004). Allocentric and egocentric updating of spatial memories. Journal of Experimental Psychology. Learning, Memory, and Cognition, 30, 142-157.

O'Keefe, J., \& Conway, D. H. (1980). On the trail of the hippocampal engram. Physiological Psychology, 8, $229-238$.

Presson, C. C. (1987). The development of landmarks in spatial memory-the role of differential experience. Journal of Experimental Child Psychology, 44, 317-334.

Presson, C. C., \& Montello, D. R. (1994). Updating after rotational and translational body movementscoordinate structure of perspective space. Perception, 23, 1447-1455.

Rieser, J. J. (1989). Access to knowledge of spatial structure at novel points of observation. Journal of Experimental Psychology. Learning Memory and Cognition, 15, 1157-1165. 
Roskos-Ewoldsen, B., McNamara, T. P., Shelton, A. L., \& Carr, W. (1998). Mental representations of large and small spatial layouts are orientation dependent. Journal of Experimental Psychology. Learning, Memory, and Cognition, 24, 215-226.

Sandstrom, N. J., Kaufman, J., \& Huettel, S. A. (1998). Males and females use different distal cues in a virtual environment navigation task. Brain Research. Cognitive Brain Research, 6, 351-360.

Scholkopf, B., \& Mallot, H. A. (1995). View-based cognitive mapping and path planning. Adaptive Behavior, 3, $311-348$.

Silverman, I., \& Eals, M. (1992). Sex differences in spatial abilities: Evolutionary theory and data. In L. Cosmides, \& J. Tooby (Eds.), The adapted mind: Evolutionary psychology and the generation of culture (pp. 487-503). New York: Oxford University Press.

Simons, D. J., \& Wang, R. F. (1998). Perceiving real-world viewpoint changes. Psychological Science, 9 , 315-320.

Suzuki, S., Augerinos, G., \& Black, A. H. (1980). Stimulus control of spatial behavior on the eight arm radial maze. Learning and Motivation, 11, 1-8.

Tlauka, M., \& Wilson, P. N. (1994). The effect of landmarks on route-learning in a computer-simulated environment. Journal of Environmental Psychology, 14, 305-313.

Waller, D., Loomis, J. M., Gollege, R. G., \& Beall, A. C. (2000). Place learning in humans: The role of distance and direction information. Spatial Cognition and Computation, 2, 333-354.

Wang, R. F., \& Simons, D. J. (1999). Active and passive scene recognition across views. Cognition, 70, $191-210$.

Wang, R. F., \& Spelke, E. (2000). Updating egocentric representations in human navigation. Cognition, 77, $215-250$.

Wang, R. F., \& Spelke, E. S. (2002). Human spatial representation: Insights from animals. Trends in Cognitive Sciences, 6, 376-382.

Winer, B. J., Brown, D. R., \& Michels, K. M. (1991). Statistical principles in experimental design (3rd ed.). New York: McGraw-Hill.

Wraga, M., Creem, S. H., \& Proffitt, D. R. (2000). Updating displays after imagined object and viewer rotations. Journal of Experimental Psychology. Learning, Memory, and Cognition, 26, 151-168. 